\title{
92
}

\section{Hypermedia for mathematics: authoring courses with HMLE}

\author{
Jari Multisilta \\ Kostadin Antchev \\ Seppo Pohjolainen \\ Tampere University of Technology \\ Finland
}

\begin{abstract}
This paper describes a hypermedia based learning environment called HMLE for authoring courses in mathematics. HMLE enables the author to use mathematical text with formulas, mathematical tool programs, concept maps, interactive exercises and digitized videos. In the paper HMLE is compared with a number of other hypermedia environments and the differences between these environments are shown. The HMLE tools are being used to create a pilot hypermedia based course on matrix algebra at Tampere University of Technology (TUT).
\end{abstract}

Main conference themes: software

Educational areas: higher education

Study topics: mathematics

Secondary keywords: authoring systems, hypermedia, thinking 


\section{INTRODUCTION}

Object oriented programming environments, such as HyperCard, have made it possible to easily integrate text, graphics, animation, mathematical programs, digitized videos and sound into hypermedia.

However, to date it has not been easy to present mathematical information in hypermedia authoring systems. These did not support use of a mathematical tool program in a hypermedia environment. Researchers have tried to remove these restrictions from HyperCard; Siviter and Brown [1] for example have developed an environment where the author can add educational activity nodes from different applications to the hypermedia. A set of tools for creating (mathematical) hyperbooks was developed by Krönefors and Lundberg [2]. Mathematical hypermedia in HyperCard were supported by a tool which can be used to create mathematical formulas. However, this tool is useful only for quite simple formulas.

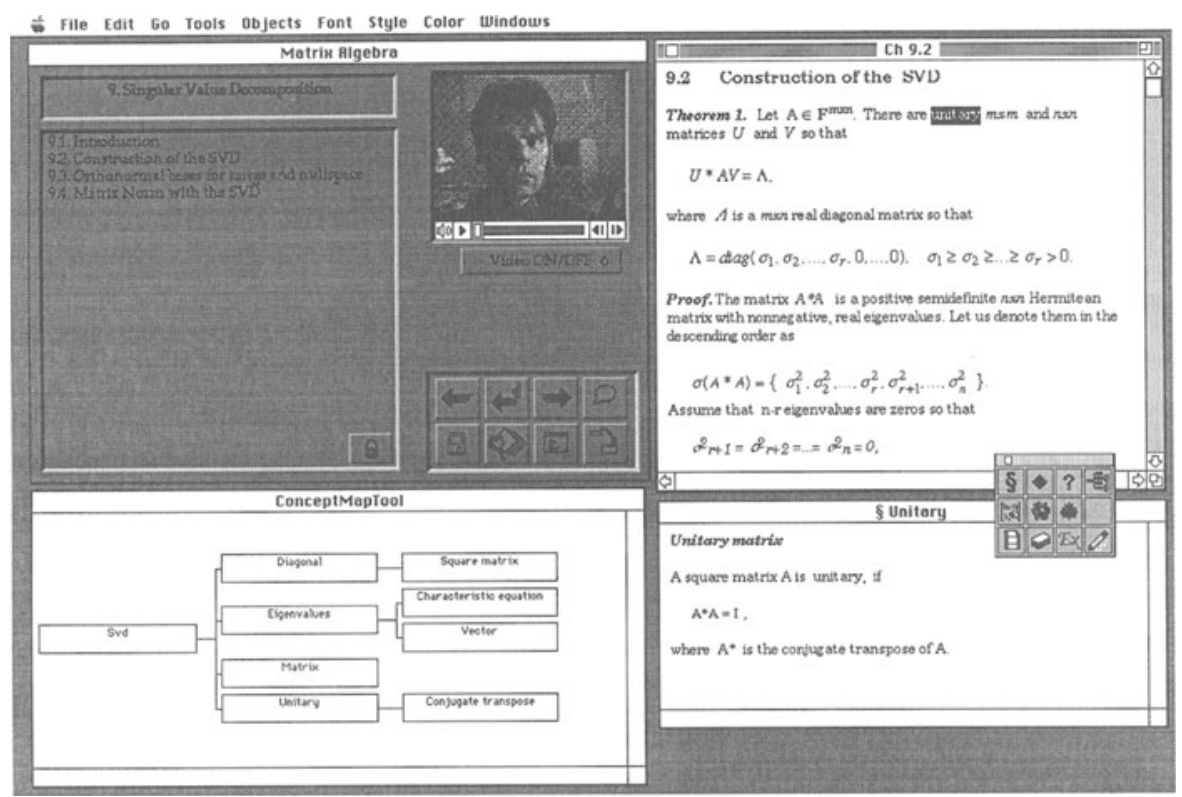

Fig. 1 An overview of HMLE for matrix algebra

We have developed the HMLE software to generate hypermedia based learning environments for mathematics learning [3, 4]. Figure 1 gives an overview of 
HMLE tools as used in a matrix algebra course application. We have not only developed a single hypermedia course on mathematics, but also a set of software tools for translating lecture notes in the mathematical sciences into hypermedia and for supporting the learning process. In this paper we demonstrate the use of these tools in the design of the Matrix Algebra course.

\section{HYPERMEDIA ENVIRONMENTS FOR MATHEMATICS EDUCATION}

\section{Requirements for mathematical hypertext}

Mathematical text should be written with a text editor which is able to handle formulas sufficiently well. The editor should be able to save the text in a format (mark-up) which can be transformed to hypertext, for example RTF (Rich Text Format) or TeX/LaTeX. Other requirements for the hypermedia system for mathematics are that it should:

- integrate hypertext, computer aided exercises, graphics, video and sound;

- implement implicit links (links generated by the computer) so that every word could be an anchor to the definition of that word (if the definition exists);

- be easily available to the students (possibly as shareware);

- be able to record the actions of the students in order to examine different study styles in hypermedia learning environments;

- support the process of learning mathematics;

- be easy to author, maintain and update.

We did not find a ready made hypermedia system with all the required properties. In the next chapter we discuss a few hypermedia systems and try to point out why it was decided to build the HMLE tool.

\section{Existing hypermedia applications}

Intermedia [5] is probably the system which meets most of the requirements outlined above, but as it runs under A/UX (Apple Computer's implementation of the Unix operating system) it cannot be considered to be a hypermedia environment for students to use on their home computers.

A popular hypermedia application is World Wide Web (WWW) and its browser application Mosaic [6]. It is based on a client-server architecture and there are WWW clients for several hardware environments. To use WWW the user needs a browser, for example NCSA Mosaic or Netscape, and a set of viewers which are used to display complex graphics, animation and sound. NCSA Mosaic and several viewers are currently also available for X-Windows, Windows and Macintosh (public domain and shareware). 
The material for a WWW server is typically written in HTML (HyperText Markup Language) and can be made available on Internet which makes WWW suitable for distance education. For the user it is quite an easy application to use, but for the author there is not yet a good graphical editor with which to build HTML files. The author has to explicitly create all the links by writing a sequence of HTML commands in a file which makes it impossible to implement implicit links. The current syntax of HTML does not provide any support for mathematical formulas, but this feature will be added later.

LinksWare for Macintosh is a commercial product which can create hypertext links between text files created with different word processors [7]. It can open files which contain mathematical text, but as a result files may be formatted differently than in the original document. For example, formulas do not appear to have proper line heights. In addition LinksWare is not able to create links to other applications. It can however create links to Apple script command files which can open an application and execute commands for that application.

Because HyperCard is part of Macintosh system software we decided to use this as authoring environment. HyperCard can be described as an object oriented programming environment where buttons, text fields and links to other programs can be implemented rather easily. Graphics, animation and sound can also be presented with HyperCard and QuickTime system extension. HyperCard does not support all of the requirements mentioned above, but it can be extended by programming XCMD's (external commands) in C or Pascal.

\section{ARCHITECTURE OF HMLE}

\section{Database}

Traditional documents may be transformed into hypertext by reflecting the document's logical markup in the hypertext and then producing links which are not evident in the document markup [8]. Microsoft Word can save the files in RTF format (Rich Text Format, Microsoft defined), a suitable document markup language for mathematical texts which we therefore used for converting existing lecture notes into hypertext. An RTF Reader XCMD was implemented [4] to extend HyperCard. This RTF reader can read RTF files, display them in a scrollable window and dispatch mouse clicks in the window's content region to HyperCard. RTF files can also be converted to HTML files using an RTF to HTML filter which is available as shareware. This makes it possible to implement a WWW server at the moment HTML syntax will be extended to support mathematical formulas. 
The HMLE database contains basically two different types of information, namely nodes and links between nodes. A node can be any file (document) in the Macintosh file system. Nodes are saved as separate files and the database contains only a reference to the actual node (file name) and some additional information (for example the creator application and the type of node). The other part of the database is link information which is kept separate from node information and actual documents. As a result it is possible to use any file in the Macintosh file system as a node in HMLE. This is in principle an implementation similar to that in Intermedia [5].

The text can be divided into nodes in many ways. In the Matrix Algebra course there are 72 subchapters, 80 definitions and several exercises and examples which contain all the material in the original lecture notes. Subchapters form a database which can be read sequentially as with original lecture notes on paper. Definitions extend the information presented in subchapters.

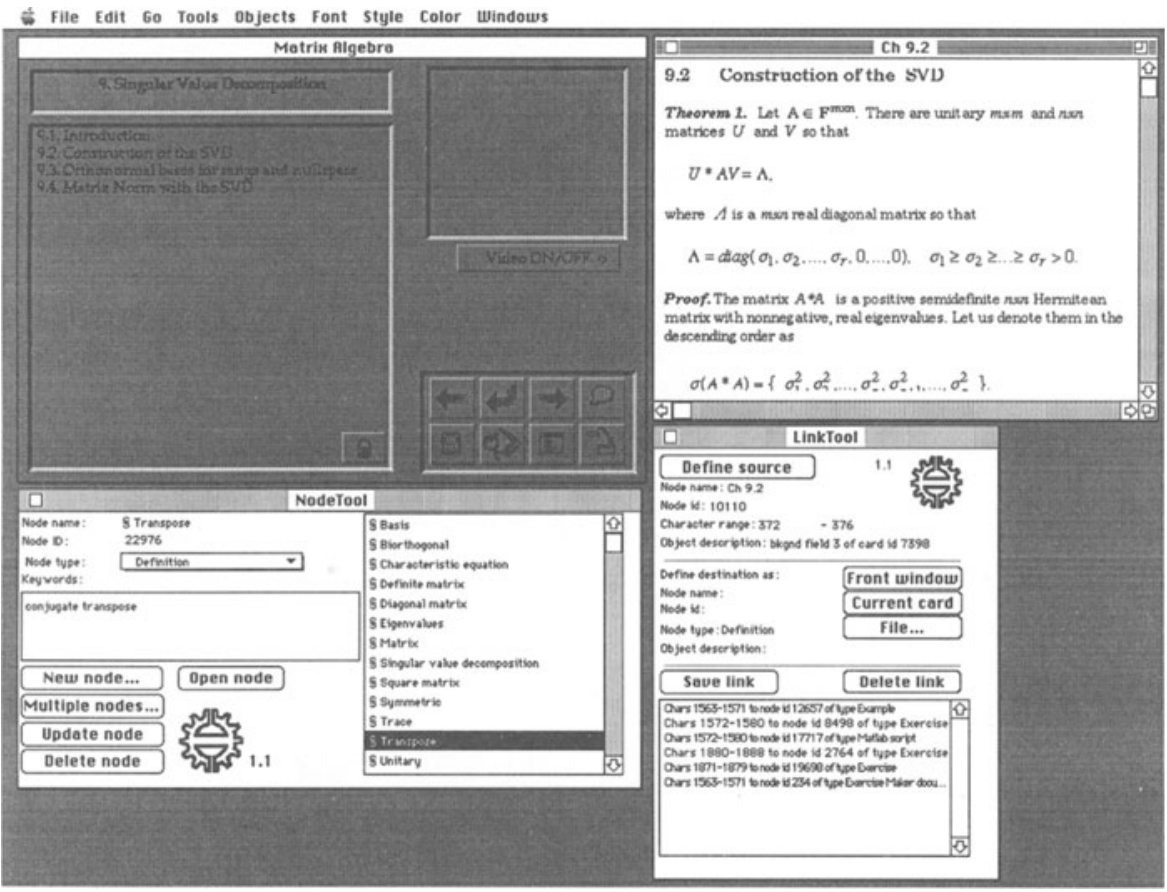

Fig. 2 Authoring tools in HMLE 


\section{Hypertext Abstract Machine (HAM)}

A Hypertext Abstract Machine, HAM [9] was needed to implement the node and link structures in hypertext. Link Tool and Node Tool (Fig. 2) were developed for this purpose [4].

There are two types of links in HMLE. On the one hand there are explicit links created by the author. With Link Tool authors define the source of the link, the destination of the link and save the link on the database. On the other hand implicit links are generated automatically by the computer. Every word in the material is considered to be a link to a predefined set of nodes containing all the definitions of mathematical concepts. Implicit links are always reference links. Explicit links can both be reference links and action links to educational activity nodes.

In the Finnish language there are many inflected forms of a word. The implicit link is a link from the inflected form of a concept to the destination node where the concept has been defined. When the actual link is generated by the computer, the inflected form is resolved to the basic form from which the destination node can be recovered.

\section{Presentation level}

Nodes in HMLE are displayed in RTF Reader, Exercise Maker or external applications. The method by which nodes are opened is determined at runtime on the basis of the information on the node contained in the database. This technique is similar to that used in the Mosaic WWW browser where some nodes can be opened by Mosaic itself and others are opened by external viewers.

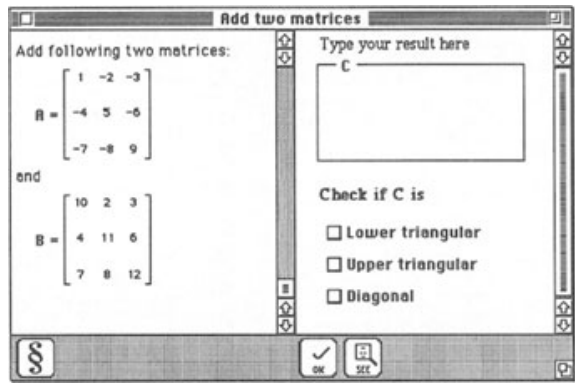

Fig. 3 Simple exercise on matrix addition

Exercise Maker provides authoring techniques and tools for setting up and presenting interactive mathematical exercises (see Fig. 3). Students can see the 
problems expressed in ordinary mathematical notation, give answers and get assistance in the form of hints and other detailed instructions. Exercise Maker is similar to The Assessor [10] which is a mathematical exercise application for Microsoft Windows. However, Assessor does not use external mathematical tools like Exercise Maker, which uses Mathematica.

An essential part of any course in applied mathematics is formed by numerical and symbolic computations with existing mathematical software. A hypermedia based learning environment should have a link to one or several of these programs. In the Matrix Algebra course Matlab is a natural companion because it supports matrix computation at all levels and offers collections of functions ('toolboxes') for many application areas.

\section{AUTHORING HMLE COURSES}

\section{The text}

Texts for hypermedia courses are written in the same way as lecture notes. Texts should be divided into subchapters, examples, exercises and definitions and saved as separate RTF files (one subchapter per file). When writing, the author does not have to create links to definitions of mathematical concepts. This will be done automatically by HMLE. However, when the author wants to create a link to an exercise or example, a graphical clue should be added into the text as a position for a link (i.e. anchor). An anchor can be an iconic character from a special font.

The next step is to convert RTF files to files which are smaller and also faster to open, with RTF Converter. Now the author can create a new course from an HMLE course template and import files by using Node Tool. In Node Tool the author defines the type and keywords for each node. The keywords form a dictionary which is used to recognize mathematical concepts from nodes and to generate concept maps (see Fig. 4).

After importing nodes the author creates a Table of Contents (TOC) by writing the titles of chapters and subchapters to TOC cards in the HMLE course template. Links from the TOC to respective subchapters are then generated with Link Tool. The author can later add explicit links and even new nodes if needed.

The authoring process in HMLE is similar to the preparation of ordinary lecture notes. The author does not have to worry about links when creating the text. Most of the links are generated by the system and the rest can be defined after the writing process is over. 


\section{Concept maps}

Concept maps have been a useful tool in helping students learn how to learn. They can be described as metacognitive tools because these transcend subject matter and assist all kinds of learners [11]. The theoretical roots of concept maps were derived from Ausubel's Assimilation Theory of Learning. In mathematics, concept maps explain which concepts students should know when studying a new one. Novak was one of the first people to use concept mapping as a tool to gain a better understanding of how new knowledge is learned and created. In Novak's method students create a concept map by first identifying key concepts and then drawing a hierarchy of concepts. Concept mapping tools similar to Novaks have been implemented for a hypermedia environment by Reader and Hammond [12]. Their tool consists of a set of drawing tools which students use to create concept maps. The Concept Map Tool implemented in HMLE differs from Reader and Hammond's tool in that concept maps are generated by the computer which means that the author does not have to do anything extra in order to include concept maps in the course.

The concept map for the Singular Value Decomposition is given in fig. 4. All the boxes may be opened to obtain the definitions of the corresponding concepts.

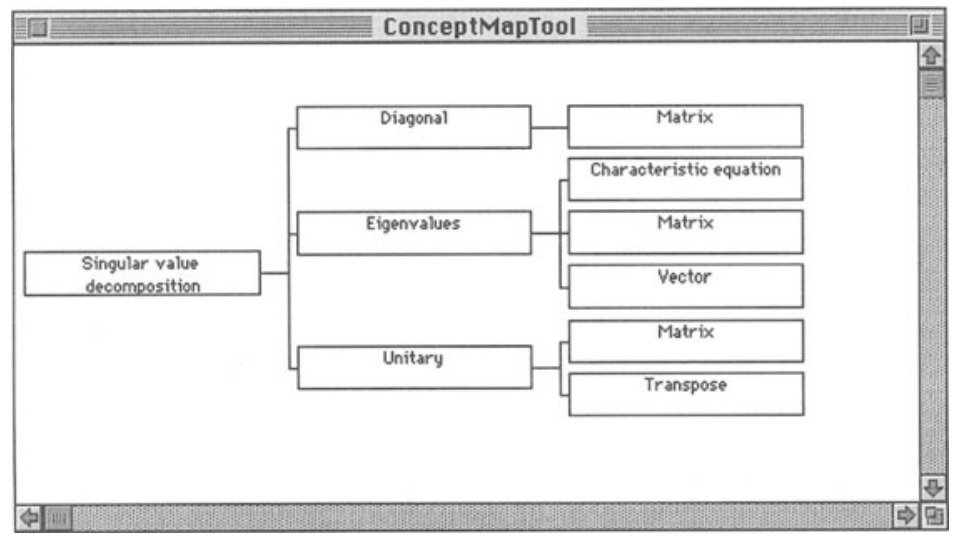

Fig. 4 Concept map for the Singular Value Decomposition.

\section{Interactive exercises}

Authoring interactive exercises for Exercise Maker needs extra effort. In addition to the description of the exercise itself the author must also write several levels of hints and possibly a solution to the exercise. All of these are written in same file, but in separate sections. The extra work involved may 
help the author to think through the solution from the student's point of view and to check whether students will be able to solve the problems.

Many exercises can of course be done using several different strategies. The author can present some of these and if students 'invent' a new solution they can be encouraged to write their own solution into the hypermedia.

A more detailed description of the implementation of Exercise Maker and authoring exercises for it is available at TUT.

Table 1 An example of exercise with two hints and a solution

Exercise: What are the requirements on the dimensions of commuting matrices?

Hint 1: Commuting matrices are such that $\mathrm{AB}=\mathrm{BA}$.

Hint 2: Use the fact that matrix multiplication is defined only when $A$ is an $(\mathrm{m} \times \mathrm{n})$ matrix and $\mathrm{B}$ is an $(\mathrm{n} \times \mathrm{p})$ matrix.

Solution: A and B must be square matrices of equal sizes.

\section{FURTHER WORK}

In order to make HMLE available to the IBM PC-environment we are implementing a similar system in ToolBook. The hypermedia material itself (RTF files, Matlab scripts and Mathematica notebooks) can be easily transferred to IBM PCs, but authoring and learning tools need to be rewritten.

The HMLE for Matrix Algebra will be used in the Matrix Algebra course at TUT this autumn. We will form a special laboratory group of 10-20 students who will take the whole Matrix Algebra course using HMLE. Teaching this group will be organized such that students use 2-3 hours a week in a computer laboratory reading the week's material from HMLE and solving selected problems on the week's topic. The teacher will be present as a consultant and formal lectures will not be given.

A hypermedia course on 'Introduction to University Level Mathematics' is also under development. 


\section{CONCLUSIONS}

In this paper the use of hypermedia in teaching mathematics has been discussed. Hypermedia is seen as a powerful framework for learning mathematics. As there have not been very many authoring tools for mathematical hypermedia we ourselves implemented a Hypermedia based Learning Environment (HMLE) for mathematics. The process of authoring a mathematical course with HMLE has been explained. It has been shown how these tools can help authors to produce a hypermedia course in a way similar to the writing original lecture notes.

\section{ACKNOWLEDGEMENTS}

The work has been supported by Finnish Academy (Suomen Akatemia), the Finnish Ministry of Education and Tampere University of Technology. Their support is gratefully acknowledged.

\section{REFERENCES}

1. Siviter D., Brown K. (1992) Hypercourseware. Computers Educ., 18 (1-3) pp. 163-170.

2. Krönefors R., Lundberg L. (1991) Hyperbooks and Authoring Tools for Hyperbooks. Proceedings of Nordic Conference on Computer Aided Higher Education, Otaniemi, Finland, pp. 188-198.

3. Multisilta J. and Pohjolainen S. (1993) Teaching Engineering Mathematics with Hypermedia. Prep. of Technology in Mathematics Teaching, University of Birmingham.

4. Multisilta J. and Pohjolainen S. (1994) Implementation of Authoring Tools for Hypermedia Based Learning Environments in Mathematics. Proceedings of CALISCE'94, Paris, France.

5. Haan B. J., Kahn P., Riley V. A., Coombs J. H. and Meyrowitz N. K. (1992) IRIS Hypermedia Services. Communications of the ACM, 35 (1).

6. Berners-Lee T., Cailliau R., Luotonen A., Nielsen H. F. and Secret A. (1994) The World-Wide-Web. Communications of the ACM, 37 (8). 
7. LinksWare Corp. (1993) LinksWare ${ }^{\mathrm{TM}}$ 3.0. LinksWare Corporation, Monterey, CA.

8. Rada R. (1992) Converting a Textbook to Hypertext. ACM Transactions on Information Systems, 10 (3).

9. Champbell B., Goodman J. M. (1988) HAM: General Purpose Hypertext Abstract Machine. Communications of the ACM, 31 (7).

10. Beilby M. (1994) Making a feature of assesment. Maths\&Stats-the newsletter of the CTI Centre for Mathematics and Statistics, 5 (2).

11. Novak, J. D. (1990) Concept Maps and Vee Diagrams: Two Metacognitive Tools to Facilitate Meaningful Learning. Instructional Science (19), Kluwer Academic Publishers, pp. 29-52.

12. Reader W. and Hammond, N. (1994) Computer-Based Tools To Support Learning From Hypertext: Concept Mapping Tools and Beyond. Computers Educ., 22 (1/2) pp. 99-106. 